\section{Macro Imaging with Digital Cameras}

Bryan Burnett* and Steven Blaauw**

*Nyoptics, Inc./Meixa Tech and ** Nyoptics, Inc

\section{Bryan@meixatech.com}

Advances in charged couple device (CCD) design, low cost processor power, cheap memory and dropping prices of digital cameras over the last few years have made the CCD digital camera an attractive alternative to the film camera for many imaging applications. This is especially true in macro imaging where it appears likely that digital cameras will replace film cameras (curiously, Long (2001), says otherwise). As will be described here, a digital camera equipped with a quality macro-zoom lens, generates images with a depth of field (e.g., Fig. 1) that greatly surpass images produced by a film camera with a comparable lens system.

One of the first things a student of photography learns is the greater the f-stop of a camera, the greater the depth of field. Unfortunately, physics

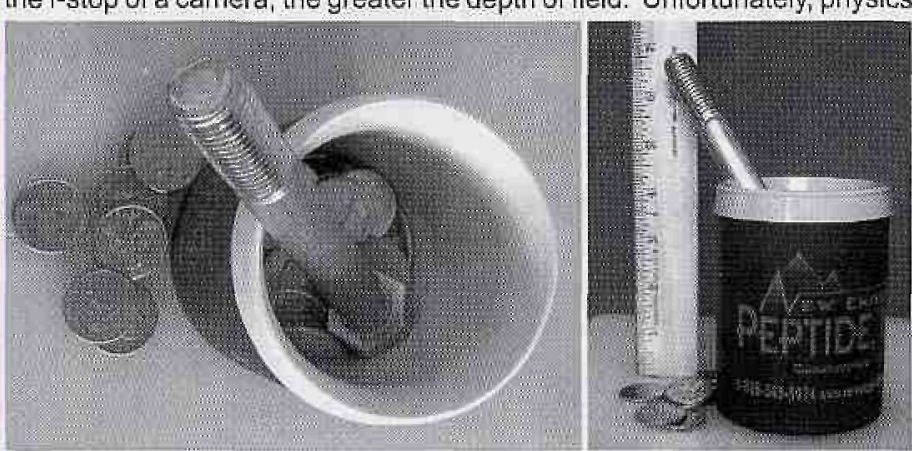

Figure 1. A: Image taken with the Pixera $600 \mathrm{CL}$ camera through the Computar MLH-10X macro zoom. B: Image taken from the side of the boit in the cup. A ruler documents the difference in elevation from the end of the bolt to the quarters on the table. Details on the US quarters as well as texture at the end of the bolt are seen despite a $174 \mathrm{~mm}$ (6-7/8 inches) difference in elevation. Image depth of field/field width equals approximately 1.

rears its ugly head; the more the f-stop increases the less amount of light that strikes the film or CCD. So, to compensate for the reduced light levels with a high f-stop, either the exposure time needs to be increased, the sensitivity of film/CCD needs to be increased, the light projected onto the specimen greatly increased, or all three. In film cameras, high film sensitivity is attained by using a high ISO-rated film. In digital cameras, sensitivity of the $C C D$ can be controlled electronically with maximum sensitivity limited by the performance of the sensor chosen. In addition, reducing the CCD sensor temperature with a Peltier cooling device provides even greater sensitivity as well as reducing the dark noise component of the chip's sensor. Software frame averaging can also be utilized to further reduce the image noise under low light conditions (an operation not practical with a film type camera). This paper examines the capabilities of a number of scientific digital cameras for producing high depth of field images.

\section{Charged coupled device (vs. CMOS)} cameras were chosen for this experiment, as the CCD cameras are superior in low light performance and image uniformity.

Materials \& Methods

The cameras used in this study are the Pixera Corporation models 120 es, 150ES, $600 \mathrm{ES}, 150 \mathrm{Cl}, 600 \mathrm{CL}$ and the Nyoptics Corporation NIC-150 (Table 1). The 120 es is a $1 / 3$-inch sensor design, while the 150 and 600 cameras use a $1 / 2$-inch sensor. The 150 series are 1.5 mega pixel cameras and the 600 series are 5.8 mega pixel with the CL models of either series having Peltier cooling. The cameras were mounted on a modified Polaroid MP4 copy stand (Fig. 2). All six models of the cameras

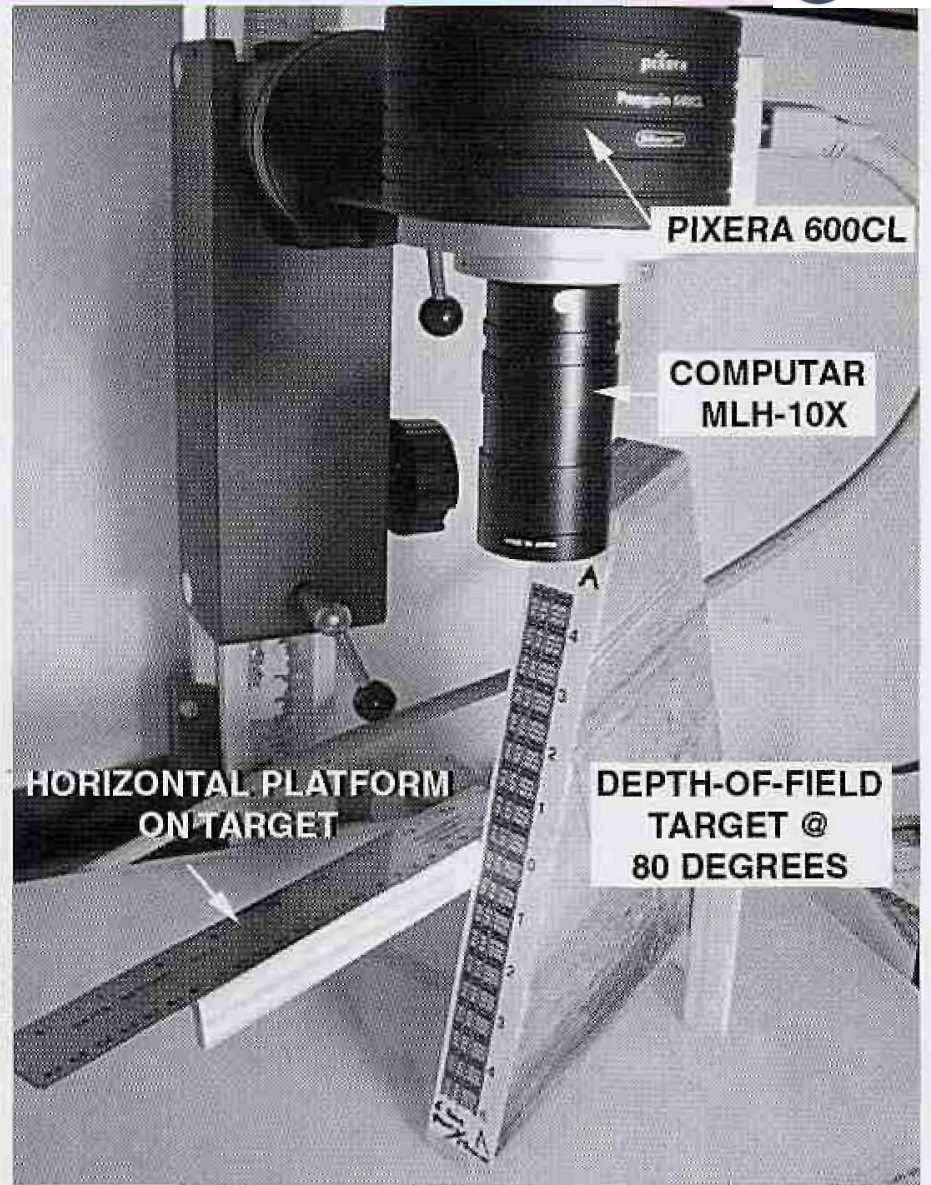

Figure 2. The apparatus for the measurement of depth of field. The camera, macro-zoom lens and the depth-of-field target are shown. The target was constructed with Adobe Photoshop and printed with a dye-sub printer. The camera is mounted on a modified Polaroid MP4 copy stand. There is a depth-of-field target available from Edmunds Scientific (edmundoptics.com, stock \#NTS 54-440), which was inadequate to test the cameras in this study, due to too low an angle (45) of the target and insufficient range (maximum depth: $50 \mathrm{~mm}$ ).

offer frame averaging within their software packages.

The macro-zoom lens used in this study is the $\mathrm{C}$-mount Computar MLH-10X Macro-zoom (f 5.6 to total closure) and is used for both $1 / 3$-and $1 / 2$ - inch CCD cameras. Noteworthy for the Computar macro-zoom is that the f-stop adjustment ring on the lens does not have defined positions for each $\mathrm{f}$-stop. One must carefully estimate the $\mathrm{f}$-stops beyond 5.6 by use of the "analog" scale provided on the Computar adjustment ring. Although the imaging results of the manually estimated position of the adjustment ring are consistent for the f-stops up to 32 (see Fig. 4), the f-stops beyond

\begin{tabular}{|c|c|c|c|c|}
\hline \multicolumn{3}{|c|}{ Table 1. Models of Pixera digital cameras tested. Sensitivity ratings provided by Pixera. } \\
\hline Model\# & $\begin{array}{c}\text { Resolution (width } x \\
\text { height) }\end{array}$ & CCD type & Chip size & Sensitivity (lux) \\
\hline $120 \mathrm{es}$ & $1260 \times 960$ & mosaic & $1 / 3^{\prime \prime}$ DiRactor & 0.3 \\
\hline $150 \mathrm{ES}$ & $1392 \times 1040$ & mosaic & $1 / 2^{\prime \prime}$ & 0.05 \\
\hline $600 \mathrm{ES}$ & $2776 \times 2074$ & mosaic & $1 / 2^{\prime \prime}$ DiRactor & 0.05 \\
\hline $150 \mathrm{CL}$ & $1392 \times 1040$ & mosaic & $1 / 2^{\prime \prime}$ & 0.01 \\
\hline $600 \mathrm{CL}$ & $2776 \times 2074$ & mosaic & $1 / 2^{\prime \prime}$ DiRactor & 0.01 \\
\hline NIC-150 & $1392 \times 1040$ & mosaic & $1 / 2^{\prime \prime}$ & 0.07 \\
\hline
\end{tabular}




\section{Piecing Together Analytical Solutions}
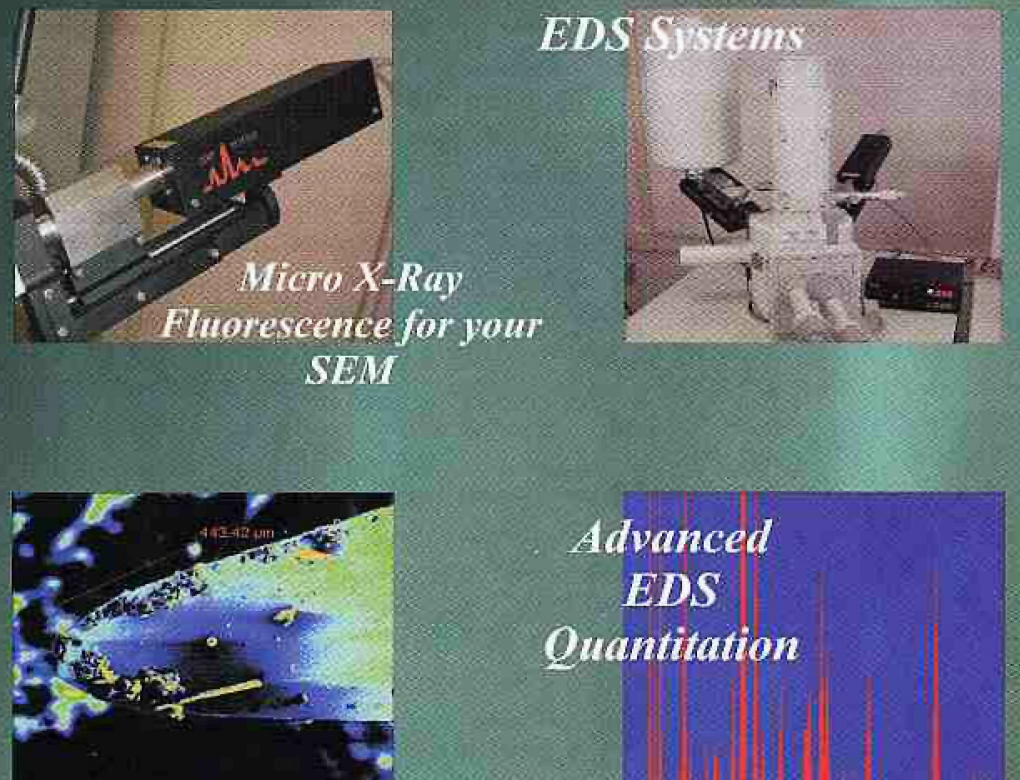

Digital Imaging and Advanced Peature Measuments

DXRF Systems, Inc. 15715 Brookford Dr. Houston, TX, 77059 USA
Whether it is Digital Image Analysis X-ray Mapping or Quantitative BDS you are looking for, IXRF has your solution. Designed to meet the analytical needs of you, the user simple yet powerful tools provide a price to performance ratio that is unparalleled.

Flexible, full customization throughout the software

- Comprehensive, adaptive spectrum processing and the highest level of quantitative analysis

Powerful, automated, and complete statistical digital image analysis that includes Feature Analysis on phases or particles

Highly interactive, showcase, fullspectrum $x$-ray mapping that includes fractionated phase or chemical composition statistical analysis

Remember: As evidence of our commitment to our customers, IXRF will provide Free software upgrades for as long as you own your system. Our software is improved and upgraded largely based on customer suggestions.

But this should be old news. Over the past decade IXIRF has fundamentally changed the industry. As another example of our dedication to innovtion, we are offering, with an industry first, the addition of small-spot XRF analysis to the world of Scanning Electron Microscopy. Experience elemental sensitivities and detection limits that are 10100 times greater than electron beam excitation. Imagine looking at a sample and seeing 5 elemental peaks. NOW, imagine "discovering" an additional 25 peaks!!

We pride ourselves in customer service. Call us at (281) 286-6485 to talk to any of our staff, or ask one of our hundreds of eustomers-after all- they' re our best sales people! 


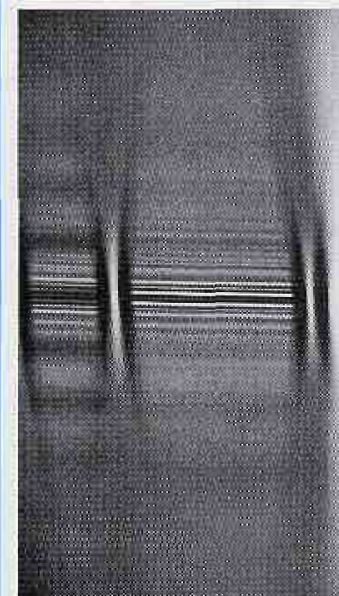

A
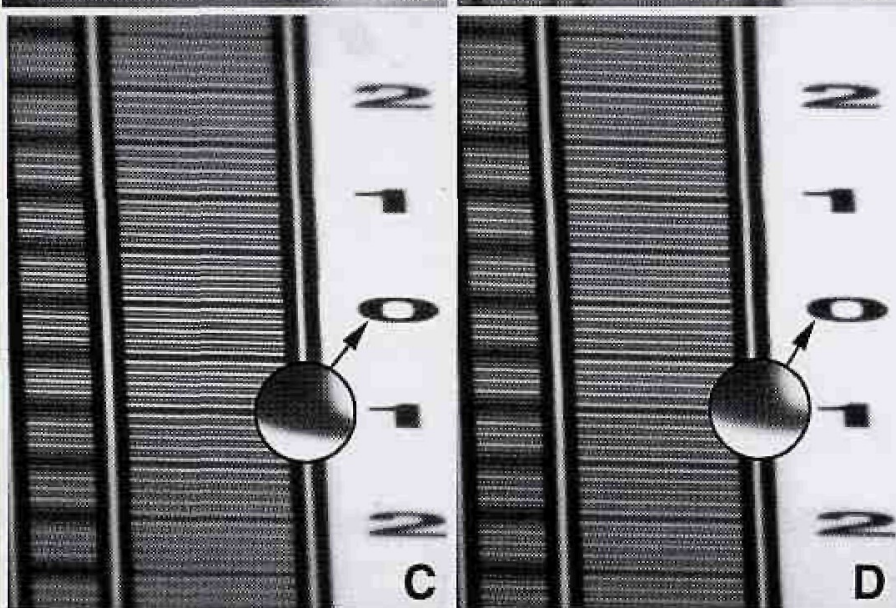

Figure 3. Images from the Pixera $600 \mathrm{CL}$ tests at the field width of $20 \mathrm{~mm}$. A: The depth of field target at $f 5.6$. B: The target image at the same position at $f$ 32. C: The target image at one $f$-stop greater than $f 32$ (2.8 sec exposure at ISO 200 setting for the camera, 10 frames averaged). $D$ : The target image at two f-stops greater than $f$ 32 (5.2 sec exposure at $1 S O 200$ setting for the camera, 10 frames averaged).

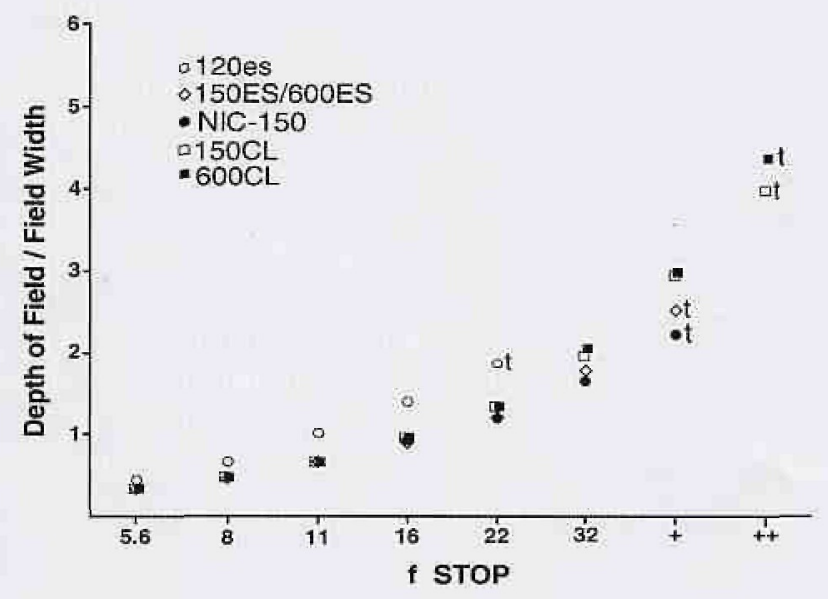

Figure 4. Graph of the f-stop vs. depth of field/field width for five different Pixera camera models with the Computar MLH-10X macro zoom lens. The $150 E S$ and the $600 E S$ points were equivalent and are plotted together. The $150 \mathrm{CL}$ and $600 \mathrm{CL}$ diverge at the second "f stop" $(++)$ beyond $f 32$. " $t$ ", indicates for each the camera, the final $f$-stop where images are poor quality (excessive graininess or blurred) when taken beyond that $f$-stop setting. The depth of fieid values presented here are based on the discernable line pairs of the depth of field target and are not indicative of the actual "usable" depth of field for imaging at that $f$-stop. See text for details.

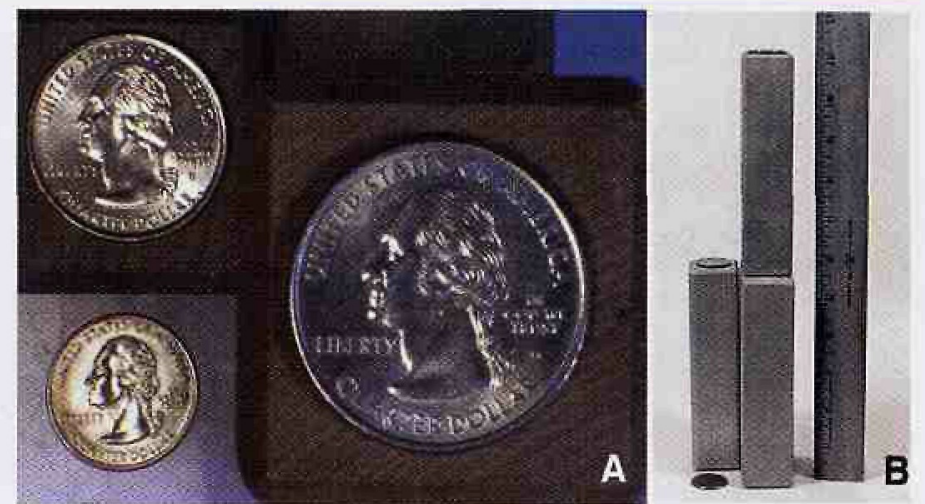

Figure 5. A: Image taken with a 600CL of US quarters on blocks of wood. The image was levels adjusted and sharpened in Adobe Photoshop. The camera was focused on the middle quarter with the Computar iris at $f 5.6$ and the image taken at an f-stop slightly above "+" (i.e., more than one f-stop above 32, see Fig. 4). Image depth of field/field width is approximately 3.5. B: Side view of the same objects shown in A. Approximately $140 \mathrm{~mm}$ (5 1/2 inches) separate each quarter for a total depth of field of more than $280 \mathrm{~mm}$ ( 11 inches).

32 had to be estimated by the exposure time provided by the camera's software. The two estimated f-stops beyond 32 are indicated by " + " and "t+" in Fig. 4.

A depth-of-field target was constructed with an $80^{\circ}$ incline (Fig. 2). There is a horizontal platform attached at the middle of the target. On the target surface there are horizontal line pairs (two black lines separated by white with a total width of $1-\mathrm{mm}$ per pair). The line pairs are separated by white space of approximately $0.8 \mathrm{~mm}$. To assist in counting, for every five line pairs, the fifth line pair is separated by red rather than white. The target range is $250 \mathrm{~mm}$. Focusing and image size adjustments were made on a ruler that rests on the horizontal platform. Final fine focus adjustments were made on the copy stand by adjusting the center of focus on the " 0 " line of the target at $f 5.6$ (Fig. 2).

Images (e.g., Fig. 3) were taken with each camera at f 5.6, 8, 11, 16, 22,32 and two $f$-stops beyond 32 if possible. The number of line pairs was then counted. A line pair was counted if a gap (white or red space) could be discerned between the lines of the line pairs.

\section{Results and Discussion}

Table 2 shows the number of identifiable line pairs at different $f$-stops for the Pixera $120 \mathrm{es}$ with a field width of $20 \mathrm{~mm}$. At $\mathrm{f} 22$ the images taken by the 120 es show a color shift to red and more noise than images captured at lower f-stops. An image-processing program, such as Adobe Photoshop, can be used to compensate for the color shift. Image quality for the $120 \mathrm{es}$ at $f 22$ at one frame is poorer than that of an image utilizing the frame averaging capture technique with an average of 20 frames. The 120 es cannot produce adequate images at $f 32$ even with frame averaging. The reason is likely that the Toshiba CCD used in the $120 \mathrm{es}$ is not as sensitive as the Sony CCD, which is used in the Pixera $150 \mathrm{ES}, 600 \mathrm{ES}, 150 \mathrm{CL}, 600 \mathrm{CL}$ and the Nyoptics NIC-150 (Table 1).

For the Pixera 120es, the Computar macro-zoom lens' focus and zoom had to be readjusted to achieve $20-\mathrm{mm}$ field width from the positions used with the $600 \mathrm{ES}$ and $600 \mathrm{CL}$. The $120 \mathrm{es}$ is able to achieve a depth of field approximately $25 \%$ greater at comparable f-stops than that achieved by the other Pixera cameras at $20 \mathrm{~mm}$ field width.

The results of the cameras tested as well as the data presented for the Pixera 120es (Table 2) are plotted in Fig. 4. Noteworthy is that these data show the increase in depth of field with f-stop is not linear, rather, the relationship appears exponential.

The difference between the Pixera ES and CL cameras are sensitivity (Table 1). The $150 \mathrm{CL}$ and $600 \mathrm{CL}$ cameras are able to generate reasonableclarity images (i.e., not blurred or grainy) at one f-stop greater than the 150 ES and 600ES. This sensitivity difference $(5 x)$ between the cooled $(C L)$ and 
un-cooled (ES) CCD makes for a substantial difference in the depth of field capabilities (Fig. 4).

The Pixera DiRactor system allows for four-fold increase of resolution, without increase in chip area, from the $150 \mathrm{ES}$ and $150 \mathrm{Cl}(1392 \times 1040)$ to the 600ES and $600 \mathrm{CL}(2776 \times 2074$ ), respectively. For the $150 \mathrm{ES}$ and $600 \mathrm{ES}$ cameras, the increase in resolution does not appear to affect the number of discernable line pairs in the depth of field target to the final usable $\mathrm{f}$-stop for this camera. For the $150 \mathrm{CL}$ and $600 \mathrm{CL}$ the resolution difference appears to make a slight difference in the highest attainable f-stop (" ++ " in Fig. 4). There is, however, a progressive decrease in image sharpness with increase in $f$-stop for images captured above $f 32$ (Fig. 3 , compare insets).

The graph plotting depth of field versus f-stop (Fig. 4), suggests that images with exceptional depth of fields can be taken with the $600 \mathrm{CL}$. The ratio of depth of field/field width for the image in Fig. 1 is approximately 1 . The graph in Fig. 4 suggests that an image with a ratio greater then 4 can be taken with the $600 \mathrm{CL}$. A test image with a ratio of 3.5 was taken with the $600 \mathrm{CL}$ (Fig. 5A). The quarter closest to the lens in Fig. $5 \mathrm{~A}$ is slightly out of focus. An increase in $\mathrm{f}$-stop in order to get the quarter in focus results in an unacceptable decrease in image sharpness. Thus, it is apparent that satisfactory images cannot be obtained with depth of field/field width ratio beyond 3.5. In other words, an image ratio of 3.5 would be about the maximum one could employ to obtain images with the Computar macro zoom lens/Pixera $600 \mathrm{CL}$ camera system.

\section{Conclusions}

The Pixera 120 es has less resolution and greater depth of field than the 150 and 600 series cameras at comparable $f$-stops. We believe that this is a result of the Computar lens relative to the CCD sensor size. As the sensor size is reduced ( $E S$ and CL cameras $=1 / 2$ inch, 120 es $=1 / 3$ inch), the resulting magnification increase requires the magnification of the zoom to be reduced to maintain the same field-of-view. The reduced zoom results in a lower numerical aperture (NA) and an increase in depth of field. (Depth of Field $=0.0005 / \mathrm{NA}^{2}$ in $\mathrm{mm}$ ). Additionally, this reduced NA value also has the effect of reducing the resolution capabilities of the lens (Resolution in line-pairs $=(3000 \times \mathrm{NA}) / \mathrm{mm})$. Another factor may be the pixel pitch for the Toshiba 1/3-inch CCD chip used in the 120 es is larger $(9.6 \mathrm{~mm} \times 7.5 \mathrm{~mm}$ ) than that of the Sony $1 / 2$ inch $\operatorname{CCD}(4.65 \mathrm{~mm} \times 4.65 \mathrm{~mm})$ that is used in the Pixera $600 \mathrm{CL} / \mathrm{ES}, 150 \mathrm{CL} / \mathrm{ES}$ and NIC-150 cameras.

The depth of field increase with f-stop is nonlinear (Fig. 4). There is a substantial decrease in image quality for the Pixera 120 es between $f 16$ and $\mathrm{f} 32$. The electronic gain needed to image at these greatly reduced light levels causes increased image noise, which cannot be compensated even by frame averaging. A similar problem occurs, of course, with film cameras in that the higher the film speed, the lower the image quality due to larger grain sizes in high ISO films. However, for the 150/600ES cameras, the high sensitivity of their CCDs allows for images to be taken at just above $\mathrm{f} 32$. Further, the even higher sensitivity of the Peltier cooled CCDs in the $150 / 600 \mathrm{CL}$ cameras allows for images to be taken at least one f-stop above 32.

Frame averaging, except for the 120 es (Table 2) had a little or no apparent effect on the discernable number of line pairs for the $150 / 600 \mathrm{ES}$ and $150 / 600 \mathrm{CL}$ cameras. Unlike the Pixera $120 \mathrm{es}$, the $150 \mathrm{ES}, 600 \mathrm{ES}, \mathrm{NIC}-150$, $150 \mathrm{CL}$ and $600 \mathrm{CL}$ did not show image color shifts at any f-stop. At one f-stop beyond $\mathrm{f} 32$ the $600 \mathrm{CL}$ shows a slight reduction in image contrast as well as a slight blurring (Fig. $3 \mathrm{C}$, inset). Two $\mathrm{f}$-stops beyond $\mathrm{f} 32$ images taken by the $600 \mathrm{CL}$ exhibit pronounced image blurring (Fig. 3D, inset).

Comparisons of the 150ES with the 600ES show that despite the higher resolution of the latter, the numbers of discernable lines on the depth of field target remain the same (Fig. 4). This relationship is not quite true for the $150 \mathrm{CL}$ and $600 \mathrm{CL}$, where there appears to be a small divergence in the

number of discernable lines at two f-stops above f 32 (Fig. 4). The NIC-150 although having a similar optic system as the $150 \mathrm{ES}$, has a slightly reduced depth of field capability from that of the $150 E S$. The higher resolution of the 600 series of cameras does allow for greater enlargement of the images.

We have shown that all the scientific digital cameras examined in this study can acquire high depth of field images with a good macro-zoom lens such as the Computar MLH-10. The Pixera $600 \mathrm{CL}$, a high resolution and cooled CCD digital camera that was manufactured for the fluorescent microscopy market has exceptional capabilities in this regard (Fig. 5A). 1. Reference cited.

Long, B. (2001). Complete digital photography. Charles River Media, Inc. Hingham. p. 427

Acknowledgement: We wish to thank Yuji ide of Pixera Corporation for his valuable assistance and comments on the manuscript.
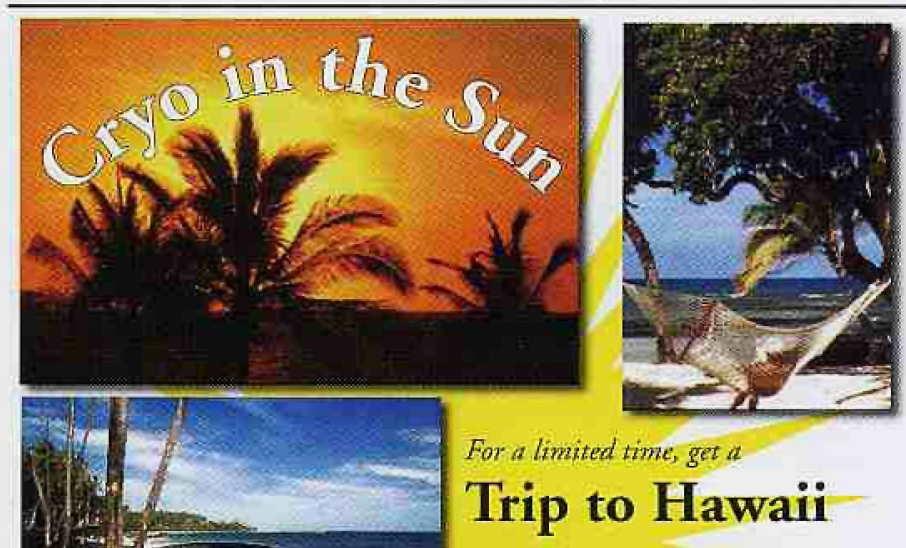

For a limited time, get $a$

Trip to Hawaii

Come See Us

Booth 1019 $M$ and $M 2003$

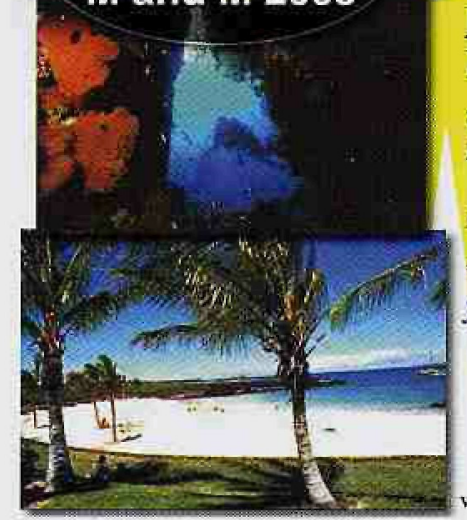

to attend

\section{Cryo in the Sun}

Just purchase a

PowerTome X or XL and $\mathbf{C R}-\mathbf{X}$ unit.

Expires 12/31/03. Sec your dealer for details.

It could be the most

enjoyable learning experience you've ever bad.

\section{RME Products}

